\title{
Stochastic Modeling of the KCLMS Algorithm in the Context of Adaptive Beamforming
}

\author{
Khaled Jamal Bakri, Eduardo Vinicius Kuhn, Marcos Vinicius Matsuo, \\ Ciro André Pitz, Rui Seara, and Jacob Benesty
}

\begin{abstract}
This paper presents a stochastic model for the Kronecker product constrained least-mean-square (KCLMS) algorithm applied in adaptive beamforming. Such an algorithm considers that the beamforming vector can be expressed as a Kronecker product of two smaller vectors, thus yielding improved convergence and less computational complexity (in comparison to the CLMS). The proposed model entails expressions describing the mean behavior of the beamforming vectors, the evolution of the output signal-to-interference-plus-noise ratio (SINR), and the correlation matrices related to the beamforming vectors. Simulation results are shown confirming the accuracy of the model.
\end{abstract}

Keywords-Adaptive beamforming, antenna arrays, CLMS algorithm, Kronecker product, mobile communications.

\section{INTRODUCTION}

Mobile communication systems have used adaptive beamforming techniques to dynamically adjust the radiation pattern of an antenna array, aiming to reduce interferences and strengthen the signal-of-interest (SOI); as a result, an improved signal-to-interference-plus-noise ratio (SINR) is achieved at the array output $[1,2]$, thus increasing both spectral and energy efficiency of the system [3]. Among the beamforming algorithms (e.g., [4-9] and references therein), the constrained least-mean-square (CLMS) [4] has been widely used, due to its low computational complexity and dependence only on knowledge of the angle-of-arrival (AOA) of the SOI [10]. However, this algorithm shows poor convergence characteristics as the number of antennas increases [7], limiting its use in modern massive MIMO (multiple-input multiple-output) applications (such as in $5 \mathrm{G}$ networks $[11,12]$ ). So, aiming to overcome this drawback, the beamforming problem has been reformulated in [13] (based on [14]) by assuming that the beamforming vector can be expressed as a Kronecker product of two smaller beamforming vectors, thus giving rise to a

Khaled Jamal Bakri and Rui Seara are with LINSE-Circuits and Signal Processing Laboratory, Department of Electrical and Electronics Engineering, Federal University of Santa Catarina, Florianópolis, SC, 88040-900, Brazil (e-mails: khaled@linse.ufsc.br; seara@linse.ufsc.br).

Eduardo Vinicius Kuhn is with LAPSE-Electronics and Signal Processing Laboratory, Department of Electronics Engineering, Federal University of Technology - Paraná, Toledo, PR, 85902-490, Brazil (e-mail: kuhn@utfpr.edu.br).

Marcos Vinicius Matsuo and Ciro André Pitz are with GEPS_Electronics and Signal Processing Group, Department of Control, Automation, and Computation Engineering, Federal University of Santa Catarina, Blumenau, SC, 89036-004, Brazil (e-mails: marcos.matsuo@ufsc.br; ciro.pitz@ufsc.br).

Jacob Benesty is with the INRS-EMT-National Institute of Scientific Research-Energy, Materials, and Telecommunications Research Center, University of Quebec, Montreal, QC, H5A-1K6, Canada (e-mail: benesty@emt.inrs.ca). joint optimization problem. Such a joint optimization problem has then been solved by using an alternating optimization strategy along with the steepest-descent method, which leads to the development of the Kronecker product CLMS (KCLMS) algorithm [13]. Simulation results (shown in [13]) have been used to confirm the robustness of the algorithm for different operating scenarios.

Considering its practical applicability and the improved performance achieved, a theoretical study of the KCLMS algorithm through a stochastic model can be useful to gain a deeper understanding of the algorithm behavior [as an alternative to the use of extensive Monte Carlo (MC) simulations]. Such a model aims to describe the algorithm behavior under different operating conditions, allowing to obtain fair performance comparisons, to establish cause-and-effect relationships, and to identify anomalous algorithm behavior [6, 9, 15-18]. Nevertheless, despite the inherent importance, the stochastic modeling of the KCLMS algorithm has not been discussed so far in the literature. In this context, focusing on adaptive beamforming applications in mobile communications, the present research work has the following goals:

i) to develop a stochastic model characterizing the behavior of the KCLMS algorithm;

ii) to derive expressions for predicting the mean behavior of the beamforming vectors, the evolution of the SINR, and some correlation-like matrices; and

iii) to verify and discuss the accuracy of the model for different operating scenarios.

The remainder of this paper is organized as follows. Section II revisits the operating scenario and the KCLMS algorithm. Section III presents the mathematical development followed to obtain the proposed model. Section IV shows simulation results for assessing the accuracy of the model. Lastly, Section V presents concluding remarks.

Throughout this paper, the adopted mathematical notation follows the standard practice of using lower-case boldface letters for vectors, upper-case boldface letters for matrices, and both italic Roman and Greek letters for scalar quantities. Superscripts $\mathrm{H}$ and $*$ stand for the Hermitian transpose of a matrix and the complex conjugate, respectively, $\mathrm{E}(\cdot)$ denotes the expected value, and $\otimes$ stands for the Kronecker product.

\section{Problem Statement}

Here, the operating scenario considered is introduced and, then, the general expressions of the KCLMS algorithm are revisited. 


\section{A. On the Operating Scenario}

Considering a scenario with $K$ single-antenna mobile terminals (co-channel users) and a base station equipped with an array of $M$ antennas, the baseband input vector (at a given time instant $n$ ) can be expressed as [10]

$$
\mathbf{x}(n)=\sum_{k=1}^{K} \mathbf{s}_{k}(n)+\mathbf{z}(n)
$$

with $\mathbf{s}_{k}(n)$ and $\mathbf{z}(n) \in \mathbb{C}^{M \times 1}$ representing, respectively, the baseband signal vector related to the $k$ th user and the complex additive white Gaussian noise with power $\sigma_{z}^{2}$ present at each antenna of the array. Then, assuming $L$ multipath components for each user, $\mathbf{s}_{k}(n)$ can be written as

$$
\mathbf{s}_{k}(n)=\sqrt{\rho_{k}} \sum_{l=1}^{L} \beta_{k, l}(n) \mathbf{d}\left(\theta_{k, l}\right)
$$

where $\rho_{k}$ denotes the transmission power of the $k$ th user, $\beta_{k, l}(n)$ models the complex envelope corresponding to the $l$ th multipath of the $k$ th user, and $\mathbf{d}\left(\theta_{k, l}\right) \in \mathbb{C}^{M \times 1}$ is the steering vector of the $l$ th multipath from the $k$ th user that arrives at the base station with AOA $\theta_{k, l}$. Note that $\mathbf{d}\left(\theta_{k, l}\right)$ depends on the array geometry (e.g., see [14, Sec. 2.1 and 6.1]).

Now, defining $\hat{\mathbf{w}}(n) \in \mathbb{C}^{M \times 1}$ as the adaptive beamforming vector, the antenna array output can be obtained as [10]

$$
y(n)=\hat{\mathbf{w}}^{\mathrm{H}}(n) \mathbf{x}(n) .
$$

As a result, the mean power of $y(n)$ becomes

$$
\mathrm{E}\left[|y(n)|^{2}\right]=\hat{\mathbf{w}}^{\mathrm{H}}(n) \mathbf{R} \hat{\mathbf{w}}(n)
$$

where

$$
\begin{aligned}
\mathbf{R} & =\mathrm{E}\left[\mathbf{x}(n) \mathbf{x}^{\mathrm{H}}(n)\right] \\
& =\sum_{k=1}^{K} \rho_{k} \mathbf{D}_{k}+\sigma_{z}^{2} \mathbf{I}_{M}
\end{aligned}
$$

is the autocorrelation matrix of the input data [5], in which $\mathbf{I}_{M}$ represents an $M \times M$ identity matrix and

$$
\mathbf{D}_{k}=\frac{1}{L} \sum_{l=1}^{L} \mathbf{d}\left(\theta_{k, l}\right) \mathbf{d}^{\mathrm{H}}\left(\theta_{k, l}\right)
$$

is the spatial autocorrelation matrix of the $k$ th user. So, using (4) and assuming in (5) the $i$ th user as the SOI, the output SINR can be expressed as

$$
\gamma(n)=\frac{\hat{\mathbf{w}}^{\mathrm{H}}(n) \mathbf{R}_{\text {soi }} \hat{\mathbf{w}}(n)}{\hat{\mathbf{w}}^{\mathrm{H}}(n) \mathbf{R}_{\mathrm{int}} \hat{\mathbf{w}}(n)}
$$

where

$$
\mathbf{R}_{\text {soi }}=\rho_{i} \mathbf{D}_{i}
$$

denotes the autocorrelation matrix of the SOI and

$$
\mathbf{R}_{\text {int }}=\sum_{\substack{k=1 \\ k \neq i}}^{K} \rho_{k} \mathbf{D}_{k}+\sigma_{z}^{2} \mathbf{I}_{M}
$$

is the autocorrelation matrix of the interference-plus-noise signal. Note that the numerator and denominator of (7) characterize, respectively, the mean power of the SOI and of the interference-plus-noise signal in the beamforming output.

\section{B. Revisiting the KCLMS Algorithm}

Instead of designing the $M$-dimensional (global) beamforming vector $\hat{\mathbf{w}}(n)$, it is assumed in the KCLMS algorithm [13] that $^{1}$

$$
\hat{\mathbf{w}}(n)=\hat{\mathbf{w}}_{1}(n) \otimes \hat{\mathbf{w}}_{2}(n)
$$

with $\hat{\mathbf{w}}_{1}(n) \in \mathbb{C}^{M_{1} \times 1}$ and $\hat{\mathbf{w}}_{2}(n) \in \mathbb{C}^{M_{2} \times 1}$ being two smaller (virtual) beamforming vectors, whose dimensions satisfy the condition $M=M_{1} M_{2}$. As a consequence, substituting (10) into (3), the antenna array output becomes

$$
y(n)=\left[\hat{\mathbf{w}}_{1}(n) \otimes \hat{\mathbf{w}}_{2}(n)\right]^{\mathrm{H}} \mathbf{x}(n)
$$

which is a bilinear form in $\hat{\mathbf{w}}_{1}(n)$ and $\hat{\mathbf{w}}_{2}(n)$ as in $[19,20]$. Then, solving a joint optimization problem by using an alternating optimization strategy (see details in [13]), an update rule for adjusting $\hat{\mathbf{w}}_{1}(n)$ is derived as

$$
\hat{\mathbf{w}}_{1}(n+1)=\hat{\mathbf{P}}_{2}(n)\left[\hat{\mathbf{w}}_{1}(n)-\mu_{1} \mathbf{x}_{2}(n) y^{*}(n)\right]+\hat{\mathbf{f}}_{2}(n)
$$

where $\mu_{1}>0$ denotes a step-size parameter,

$$
\begin{gathered}
\hat{\mathbf{P}}_{2}(n)=\mathbf{I}_{M_{1}}-\hat{\mathbf{C}}_{2}(n)\left[\hat{\mathbf{C}}_{2}^{\mathrm{H}}(n) \hat{\mathbf{C}}_{2}(n)\right]^{-1} \hat{\mathbf{C}}_{2}^{\mathrm{H}}(n) \\
\hat{\mathbf{C}}_{2}(n)=\left[\mathbf{I}_{M_{1}} \otimes \hat{\mathbf{w}}_{2}(n)\right]^{\mathrm{H}} \mathbf{C} \\
\mathbf{x}_{2}(n)=\left[\mathbf{I}_{M_{1}} \otimes \hat{\mathbf{w}}_{2}(n)\right]^{\mathrm{H}} \mathbf{x}(n)
\end{gathered}
$$

and

$$
\hat{\mathbf{f}}_{2}(n)=\hat{\mathbf{C}}_{2}(n)\left[\hat{\mathbf{C}}_{2}^{\mathrm{H}}(n) \hat{\mathbf{C}}_{2}(n)\right]^{-1} \mathbf{f} .
$$

In turn, for updating $\hat{\mathbf{w}}_{2}(n)$,

$$
\hat{\mathbf{w}}_{2}(n+1)=\hat{\mathbf{P}}_{1}(n)\left[\hat{\mathbf{w}}_{2}(n)-\mu_{2} \mathbf{x}_{1}(n) y^{*}(n)\right]+\hat{\mathbf{f}}_{1}(n)
$$

where $\mu_{2}>0$ represents a step-size parameter,

$$
\begin{gathered}
\hat{\mathbf{P}}_{1}(n)=\mathbf{I}_{M_{2}}-\hat{\mathbf{C}}_{1}(n)\left[\hat{\mathbf{C}}_{1}^{\mathrm{H}}(n) \hat{\mathbf{C}}_{1}(n)\right]^{-1} \hat{\mathbf{C}}_{1}^{\mathrm{H}}(n) \\
\hat{\mathbf{C}}_{1}(n)=\left[\hat{\mathbf{w}}_{1}(n) \otimes \mathbf{I}_{M_{2}}\right]^{\mathrm{H}} \mathbf{C} \\
\mathbf{x}_{1}(n)=\left[\hat{\mathbf{w}}_{1}(n) \otimes \mathbf{I}_{M_{2}}\right]^{\mathrm{H}} \mathbf{x}(n)
\end{gathered}
$$

and

$$
\hat{\mathbf{f}}_{1}(n)=\hat{\mathbf{C}}_{1}(n)\left[\hat{\mathbf{C}}_{1}^{\mathrm{H}}(n) \hat{\mathbf{C}}_{1}(n)\right]^{-1} \mathbf{f} .
$$

Note that $\mathbf{C} \in \mathbb{C}^{M \times P}$ in (14) and (19) denotes a constraint matrix, while $\mathbf{f} \in \mathbb{C}^{P \times 1}$ in (16) and (21) defines a vector containing the (fixed) gain assigned to each of the $P$ constraints (as in [4]). Lastly, from $\hat{\mathbf{w}}_{1}(n+1)$ and $\hat{\mathbf{w}}_{2}(n+1)$, the beamforming vector is updated as follows

$$
\hat{\mathbf{w}}(n+1)=\hat{\mathbf{w}}_{1}(n+1) \otimes \hat{\mathbf{w}}_{2}(n+1) .
$$

Therefore, the KCLMS algorithm has been completely characterized through (11)-(22).

\footnotetext{
${ }^{1}$ The decomposition given in (10) holds for antenna arrays with different geometries [14, Ch. 1].
} 


\section{PRoposed MOdEL}

In this section, the stochastic model characterizing the behavior of the KCLMS algorithm is derived, entailing expressions describing the mean behavior of the beamforming vectors, the evolution of the SINR, as well as recursive expressions for computing some required correlation-like matrices. To this end, the following assumptions are stated:

A1) The adaptive weight vectors $\hat{\mathbf{w}}_{1}(n), \hat{\mathbf{w}}_{2}(n)$, and $\hat{\mathbf{w}}(n)$ as well as the input vectors $\mathbf{x}_{1}(n), \mathbf{x}_{2}(n)$, and $\mathbf{x}(n)$ are assumed statistically independent of each other.

A2) The AOA of the SOI is assumed known a priori and fixed during the whole adaptation process; hence, $\mathbf{C}$ and $\mathbf{f}$ are constant.

Note that some simplifying assumptions are usually required to make the development mathematically tractable (see details in [21] and [22]).

\section{A. Mean Behavior of the Beamforming Vectors}

An expression describing the mean behavior of the beamforming vector $\hat{\mathbf{w}}_{1}(n)$ can be obtained by substituting (11) into (12), using (15), taking the expected value of both sides of the resulting expression, and considering Assumption A1); thus,

$$
\begin{aligned}
& \mathrm{E}\left[\hat{\mathbf{w}}_{1}(n+1)\right]= \\
& \left.\quad \mathrm{E}\left[\hat{\mathbf{P}}_{2}(n)\right]\left\{\mathbf{I}_{M_{1}}-\mu_{1} \hat{\mathbf{R}}_{2}(n)\right]\right\} \mathrm{E}\left[\hat{\mathbf{w}}_{1}(n)\right]+\mathrm{E}\left[\hat{\mathbf{f}}_{2}(n)\right]
\end{aligned}
$$

where

$$
\begin{gathered}
\mathrm{E}\left[\hat{\mathbf{P}}_{2}(n)\right] \cong \\
\mathbf{I}_{M_{1}}-\mathrm{E}\left[\hat{\mathbf{C}}_{2}(n)\right]\left\{\mathbf{C}^{\mathrm{H}}\left[\mathbf{I}_{M_{1}} \otimes \hat{\mathbf{K}}_{2}(n)\right] \mathbf{C}\right\}^{-1} \mathrm{E}\left[\hat{\mathbf{C}}_{2}^{\mathrm{H}}(n)\right] \\
\quad \mathrm{E}\left[\hat{\mathbf{C}}_{2}(n)\right]=\left\{\mathbf{I}_{M_{1}} \otimes \mathrm{E}\left[\hat{\mathbf{w}}_{2}^{\mathrm{H}}(n)\right]\right\} \mathbf{C} \\
\hat{\mathbf{R}}_{2}(n) \cong\left\{\mathbf{I}_{M_{1}} \otimes \mathrm{E}\left[\hat{\mathbf{w}}_{2}^{\mathrm{H}}(n)\right]\right\} \mathbf{R}\left\{\mathbf{I}_{M_{1}} \otimes \mathrm{E}\left[\hat{\mathbf{w}}_{2}(n)\right]\right\}
\end{gathered}
$$

and

$$
\mathrm{E}\left[\hat{\mathbf{f}}_{2}(n)\right] \cong \mathrm{E}\left[\hat{\mathbf{C}}_{2}(n)\right]\left\{\mathbf{C}^{\mathrm{H}}\left[\mathbf{I}_{M_{1}} \otimes \hat{\mathbf{K}}_{2}(n)\right] \mathbf{C}\right\}^{-1} \mathbf{f}
$$

with

$$
\hat{\mathbf{K}}_{2}(n)=\mathrm{E}\left[\hat{\mathbf{w}}_{2}(n) \hat{\mathbf{w}}_{2}^{\mathrm{H}}(n)\right]
$$

denoting the correlation-like matrix of $\hat{\mathbf{w}}_{2}(n)$. Similarly, from (11), (17), and (20), the mean behavior of the beamforming vector $\hat{\mathbf{w}}_{2}(n)$ can be described by

$$
\begin{aligned}
& \mathrm{E}\left[\hat{\mathbf{w}}_{2}(n+1)\right]= \\
& \left.\quad \mathrm{E}\left[\hat{\mathbf{P}}_{1}(n)\right]\left\{\mathbf{I}_{M_{2}}-\mu_{2} \hat{\mathbf{R}}_{1}(n)\right]\right\} \mathrm{E}\left[\hat{\mathbf{w}}_{2}(n)\right]+\mathrm{E}\left[\hat{\mathbf{f}}_{1}(n)\right]
\end{aligned}
$$

with

$$
\begin{gathered}
\mathrm{E}\left[\hat{\mathbf{P}}_{1}(n)\right] \cong \\
\mathbf{I}_{M_{2}}-\mathrm{E}\left[\hat{\mathbf{C}}_{1}(n)\right]\left\{\mathbf{C}^{\mathrm{H}}\left[\hat{\mathbf{K}}_{1}(n) \otimes \mathbf{I}_{M_{2}}\right] \mathbf{C}\right\}^{-1} \mathrm{E}\left[\hat{\mathbf{C}}_{1}^{\mathrm{H}}(n)\right] \\
\quad \mathrm{E}\left[\hat{\mathbf{C}}_{1}(n)\right]=\left\{\mathrm{E}\left[\hat{\mathbf{w}}_{1}^{\mathrm{H}}(n)\right] \otimes \mathbf{I}_{M_{2}}\right\} \mathbf{C} \\
\hat{\mathbf{R}}_{1}(n) \cong \\
\left\{\mathrm{E}\left[\hat{\mathbf{w}}_{1}^{\mathrm{H}}(n)\right] \otimes \mathbf{I}_{M_{2}}\right\} \mathbf{R}\left\{\mathrm{E}\left[\hat{\mathbf{w}}_{1}(n)\right] \otimes \mathbf{I}_{M_{2}}\right\}
\end{gathered}
$$

and

$$
\mathrm{E}\left[\hat{\mathbf{f}}_{1}(n)\right] \cong \mathrm{E}\left[\hat{\mathbf{C}}_{1}(n)\right]\left\{\mathbf{C}^{\mathrm{H}}\left[\hat{\mathbf{K}}_{1}(n) \otimes \mathbf{I}_{M_{2}}\right] \mathbf{C}\right\}^{-1} \mathbf{f}
$$

where

$$
\hat{\mathbf{K}}_{1}(n)=\mathrm{E}\left[\hat{\mathbf{w}}_{1}(n) \hat{\mathbf{w}}_{1}^{\mathrm{H}}(n)\right]
$$

represents the correlation-like matrix of $\hat{\mathbf{w}}_{1}(n)$. Finally, the mean behavior of the beamforming vector $\hat{\mathbf{w}}(n)$ can be determined by taking the expected value of both sides of (22) and considering Assumption A1), which yields

$$
\mathrm{E}[\hat{\mathbf{w}}(n+1)] \cong \mathrm{E}\left[\hat{\mathbf{w}}_{1}(n+1)\right] \otimes \mathrm{E}\left[\hat{\mathbf{w}}_{2}(n+1)\right] .
$$

Therefore, assuming that expressions characterizing (28) and (34) are known, the mean behavior of the beamforming vectors $\hat{\mathbf{w}}_{1}(n), \hat{\mathbf{w}}_{2}(n)$, and $\hat{\mathbf{w}}(n)$ can be predicted through (23), (29), and (35), respectively.

\section{B. Evolution of SINR}

Taking the expected value of both sides of (7), assuming that the numerator varies slowly with respect to the denominator [due to the constraint on $\hat{\mathbf{w}}(n)$ ensuring fixed gain towards the $\mathrm{SOI}$ ] in such a way that the averaging principle (AP) [23] can be invoked, and using the cyclic property of the trace operator [24], an approximate expression characterizing the SINR is obtained as

$$
\begin{aligned}
\mathrm{E}[\gamma(n)] & \cong \frac{\mathrm{E}\left[\hat{\mathbf{w}}^{\mathrm{H}}(n) \mathbf{R}_{\text {soi }} \hat{\mathbf{w}}(n)\right]}{\mathrm{E}\left[\hat{\mathbf{w}}^{\mathrm{H}}(n) \mathbf{R}_{\text {int }} \hat{\mathbf{w}}(n)\right]} \\
& =\frac{\operatorname{tr}\left[\hat{\mathbf{K}}(n) \mathbf{R}_{\text {soi }}(n)\right]}{\operatorname{tr}\left[\hat{\mathbf{K}}(n) \mathbf{R}_{\text {int }}(n)\right]}
\end{aligned}
$$

where

$$
\hat{\mathbf{K}}(n)=\mathrm{E}\left[\hat{\mathbf{w}}(n) \hat{\mathbf{w}}^{\mathrm{H}}(n)\right]
$$

is the correlation-like matrix of $\hat{\mathbf{w}}(n)$. So, the evolution of the SINR can be predicted if $\hat{\mathbf{K}}(n)$ is known.

\section{Correlation-like Matrices}

A recursive expression characterizing the evolution of $\hat{\mathbf{K}}_{1}(n)$ can be derived by substituting (11) into (12), calculating $\hat{\mathbf{w}}_{1}(n+1) \hat{\mathbf{w}}_{1}^{\mathrm{H}}(n+1)$, taking the expected value of both sides of the resulting expression, using the Assumption A1), and approximating

$$
\mathrm{E}\left[\hat{\mathbf{f}}_{2}(n) \hat{\mathbf{f}}_{2}^{\mathrm{H}}(n)\right] \cong \mathrm{E}\left[\hat{\mathbf{f}}_{2}(n)\right] \mathrm{E}\left[\hat{\mathbf{f}}_{2}^{\mathrm{H}}(n)\right] .
$$

Thereby, we have

$$
\begin{aligned}
& \hat{\mathbf{K}}_{1}(n+1) \cong \\
& \quad \mathrm{E}\left[\hat{\mathbf{P}}_{2}(n)\right]\left[\hat{\mathbf{K}}_{1}(n)-\mu_{1} \hat{\mathbf{K}}_{1}(n) \hat{\mathbf{R}}_{2}(n)-\mu_{1} \hat{\mathbf{R}}_{2}(n) \hat{\mathbf{K}}_{1}(n)\right. \\
& \left.\quad+\mu_{1}^{2}\left\{\mathbf{I}_{M_{1}} \otimes \mathrm{E}\left[\hat{\mathbf{w}}_{2}^{\mathrm{H}}(n)\right]\right\} \hat{\mathbf{R}}_{3}(n)\left\{\mathbf{I}_{M_{1}} \otimes \mathrm{E}\left[\hat{\mathbf{w}}_{2}(n)\right]\right\}\right] \mathrm{E}\left[\hat{\mathbf{P}}_{2}^{\mathrm{H}}(n)\right] \\
& \quad+\mathrm{E}\left[\hat{\mathbf{P}}_{2}(n)\right]\left[\mathbf{I}_{M_{1}}-\mu_{1} \hat{\mathbf{R}}_{2}(n)\right] \mathrm{E}\left[\hat{\mathbf{w}}_{1}(n)\right] \mathrm{E}\left[\hat{\mathbf{f}}_{2}^{\mathrm{H}}(n)\right] \\
& \quad+\mathrm{E}\left[\hat{\mathbf{f}}_{2}(n)\right] \mathrm{E}\left[\hat{\mathbf{w}}_{1}^{\mathrm{H}}(n)\right]\left[\mathbf{I}_{M_{1}}-\mu_{1} \hat{\mathbf{R}}_{2}(n)\right] \mathrm{E}\left[\hat{\mathbf{P}}_{2}^{\mathrm{H}}(n)\right] \\
& \quad+\mathrm{E}\left[\hat{\mathbf{f}}_{2}(n)\right] \mathrm{E}\left[\hat{\mathbf{f}}_{2}^{\mathrm{H}}(n)\right]
\end{aligned}
$$

with

$$
\hat{\mathbf{R}}_{3}(n)=\mathrm{E}\left[\mathbf{x}(n) \mathbf{x}^{\mathrm{H}}(n) \hat{\mathbf{w}}(n) \hat{\mathbf{w}}^{\mathrm{H}}(n) \mathbf{x}(n) \mathbf{x}^{\mathrm{H}}(n)\right] .
$$

In turn, substituting (11) into (17), determining the outer product $\hat{\mathbf{w}}_{2}(n+1) \hat{\mathbf{w}}_{2}^{\mathrm{H}}(n+1)$, taking the expected value of 
both sides of the resulting expression, using Assumption A1), and assuming

$$
\mathrm{E}\left[\hat{\mathbf{f}}_{1}(n) \hat{\mathbf{f}}_{1}^{\mathrm{H}}(n)\right] \cong \mathrm{E}\left[\hat{\mathbf{f}}_{1}(n)\right] \mathrm{E}\left[\hat{\mathbf{f}}_{1}^{\mathrm{H}}(n)\right]
$$

a recursive expression characterizing the evolution of $\hat{\mathbf{K}}_{2}(n)$ is derived as

$$
\begin{aligned}
& \hat{\mathbf{K}}_{2}(n+1) \cong \\
& \quad \mathrm{E}\left[\hat{\mathbf{P}}_{1}(n)\right]\left[\hat{\mathbf{K}}_{2}(n)-\mu_{2} \hat{\mathbf{K}}_{2}(n) \hat{\mathbf{R}}_{1}(n)-\mu_{2} \hat{\mathbf{R}}_{1}(n) \hat{\mathbf{K}}_{2}(n)\right. \\
& \left.\quad+\mu_{2}^{2}\left\{\mathrm{E}\left[\hat{\mathbf{w}}_{1}^{\mathrm{H}}(n)\right] \otimes \mathbf{I}_{M_{2}}\right\} \hat{\mathbf{R}}_{3}(n)\left\{\mathrm{E}\left[\hat{\mathbf{w}}_{1}(n)\right] \otimes \mathbf{I}_{M_{2}}\right\}\right] \mathrm{E}\left[\hat{\mathbf{P}}_{1}^{\mathrm{H}}(n)\right] \\
& \quad+\mathrm{E}\left[\hat{\mathbf{P}}_{1}(n)\right]\left[\mathbf{I}_{M_{2}}-\mu_{2} \hat{\mathbf{R}}_{1}(n)\right] \mathrm{E}\left[\hat{\mathbf{w}}_{2}(n)\right] \mathrm{E}\left[\hat{\mathbf{f}}_{1}^{\mathrm{H}}(n)\right] \\
& \quad+\mathrm{E}\left[\hat{\mathbf{f}}_{1}(n)\right] \mathrm{E}\left[\hat{\mathbf{w}}_{2}^{\mathrm{H}}(n)\right]\left[\mathbf{I}_{M_{2}}-\mu_{2} \hat{\mathbf{R}}_{1}(n)\right] \mathrm{E}\left[\hat{\mathbf{P}}_{1}^{\mathrm{H}}(n)\right] \\
& \quad+\mathrm{E}\left[\hat{\mathbf{f}}_{1}(n)\right] \mathrm{E}\left[\hat{\mathbf{f}}_{1}^{\mathrm{H}}(n)\right] .
\end{aligned}
$$

Note that the factorization theorem of a fourth-order moment of complex Gaussian variables [21,22] can be considered to rewrite (40) as

$$
\hat{\mathbf{R}}_{3}(n) \cong \mathbf{R} \hat{\mathbf{K}}(n) \mathbf{R}+\mathbf{R} \operatorname{tr}[\mathbf{R} \hat{\mathbf{K}}(n)] .
$$

Finally, a recursive expression characterizing the correlationlike matrix of $\hat{\mathbf{w}}(n)$ is obtained by calculating the outer product $\hat{\mathbf{w}}(n+1) \hat{\mathbf{w}}^{\mathrm{H}}(n+1)$ from (22), taking the expected value, and considering Assumption A1); thereby,

$$
\hat{\mathbf{K}}(n+1) \cong \hat{\mathbf{K}}_{1}(n+1) \otimes \hat{\mathbf{K}}_{2}(n+1) .
$$

Therefore, given the initial conditions $\hat{\mathbf{w}}_{1}(0)$ and $\hat{\mathbf{w}}_{2}(0)$, the mean behavior of the beamforming vectors can be predicted from (23), (29), and (35), while the evolution of the SINR can be determined through (36). So, the behavior of the KCLMS algorithm has now been completely characterized.

\section{Simulation Results}

Here, the accuracy of the model is verified by comparing MC simulation results (50 independent runs) with model predictions. To this end, we consider a base station equipped with a uniform linear array, having $M$ omnidirectional antennas spaced by a half wavelength. The scenario is formed by a SOI located at $30^{\circ}$ and six interfering signals located at $\left\{-60^{\circ}\right.$, $\left.-50^{\circ},-30^{\circ}, 0^{\circ}, 40^{\circ}, 70^{\circ}\right\}$. The signal transmitted by each user travels through $L=12$ independent fading paths with azimuth spread of $\Delta \theta_{k}$ around the mean AOA $\theta_{k}$. The signal-to-noise ratio (SNR) is $30 \mathrm{~dB}$ (related to the SOI power). The step-size values are $\mu_{1}=\mu_{2}=0.001$, while the beamforming vectors are initialized as $\hat{\mathbf{w}}_{1}(0)=\left[\begin{array}{llll}1 & 0 & \ldots & 0\end{array}\right]^{\mathrm{T}}$ with $M_{1}=4$ and $\hat{\mathbf{w}}_{2}(0)=\left[\begin{array}{llll}1 & 0 & \ldots & 0\end{array}\right]^{\mathrm{T}}$ for $M_{2}=M / M_{1}$; as a consequence, an omnidirectional radiation pattern is observed at the beginning of the adaptation process [5], since $\hat{\mathbf{w}}(0)=\hat{\mathbf{w}}_{1}(0) \otimes \hat{\mathbf{w}}_{2}(0)$. For the sake of simplicity, we consider only one constraint $(P=1)$ ensuring unity gain towards the SOI; thereby, $\mathbf{C}=$ $\mathbf{d}\left(\theta_{i}\right)$ and $\mathbf{f}=1$.

\section{A. Example 1}

This example aims to verify the accuracy of the proposed model vis-à-vis an array with $M=32$ antennas (as discussed in [25] for the IEEE 802.11ad standard), different values of

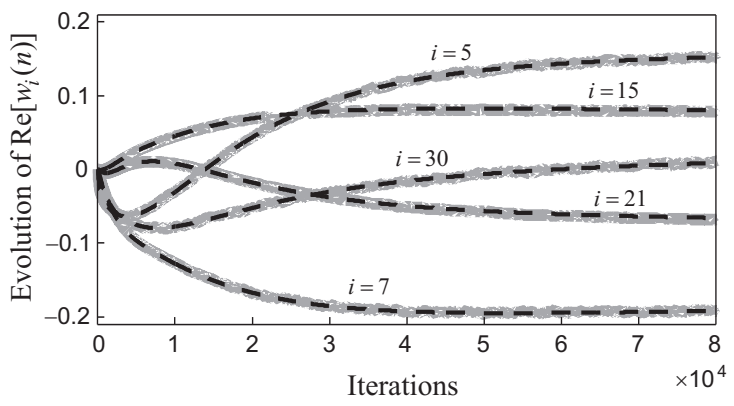

(a)

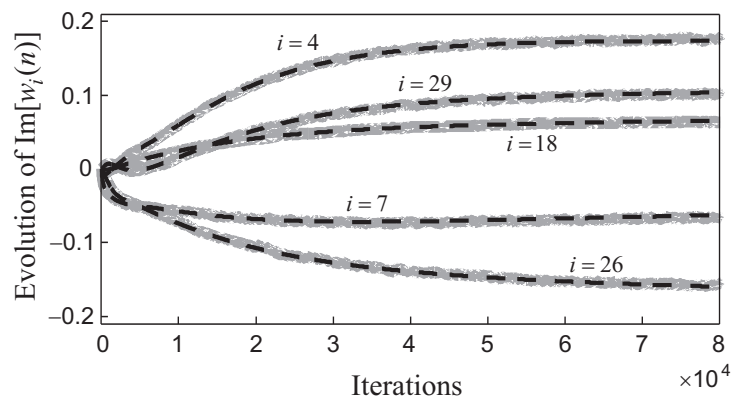

(b)

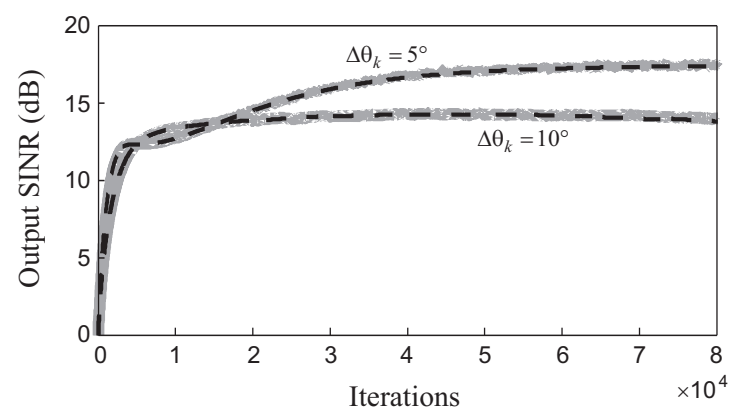

(c)

Fig. 1. Example 1. Results obtained by MC simulations (gray lines) and from the proposed model (dark-dashed lines). (a) Evolution of the real part of five weights of the beamforming vector. (b) Evolution of the imaginary part of five weights of the beamforming vector. (c) Evolution of the SINR [The case of $\Delta \theta_{k}=10^{\circ}$ leads to similar results and has been omitted in (a) and (b).]

$\Delta \theta_{k}$ (i.e., $\Delta \theta_{k}=5^{\circ}$ and $\Delta \theta_{k}=10^{\circ}$ for all $k$ ), and an input SINR $^{2}$ of $0 \mathrm{~dB}$.

\section{B. Example 2}

This example assess the accuracy of the proposed model considering an array with $M=128$ antennas (as discussed in [11] for very large MIMO systems), different input SINR values (i.e., $-7.8 \mathrm{~dB}$ and $0 \mathrm{~dB}$ ), while the azimuth spread is made equal to $\Delta \theta_{k}=5^{\circ}$.

Figs. 1 and 2 show the results obtained from MC simulations and model predictions considering the scenarios described in Examples 1 and 2, respectively. Specifically, Figs. 1(a) and 2(a) depict (for the sake of clarity) the evolution of the real part of five adaptive weights, Figs. 1(b) and 2(b) present the evolution of the imaginary part of five adaptive weights, while Figs. 1(c) and 2(c) illustrate the evolution of

\footnotetext{
${ }^{2}$ The input SINR is defined as the ratio between the SOI power and the
} interference-plus-noise power at the input of the antenna array. 


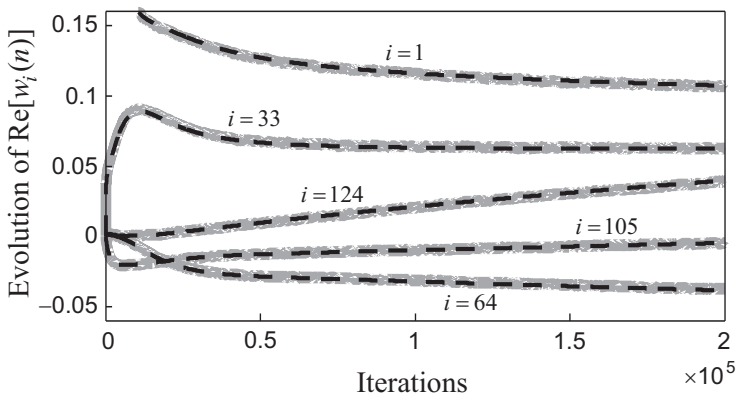

(a)

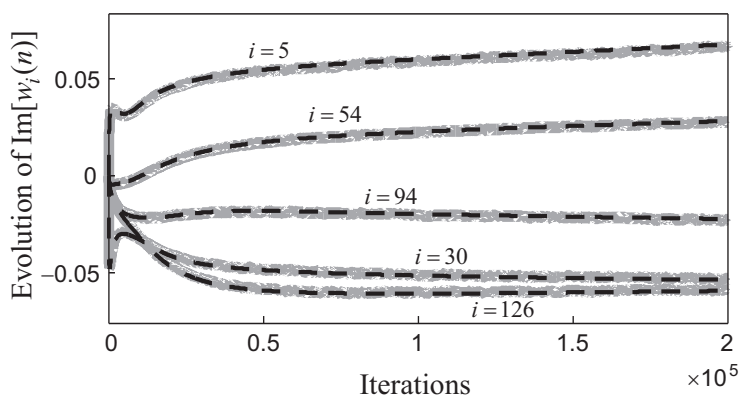

(b)

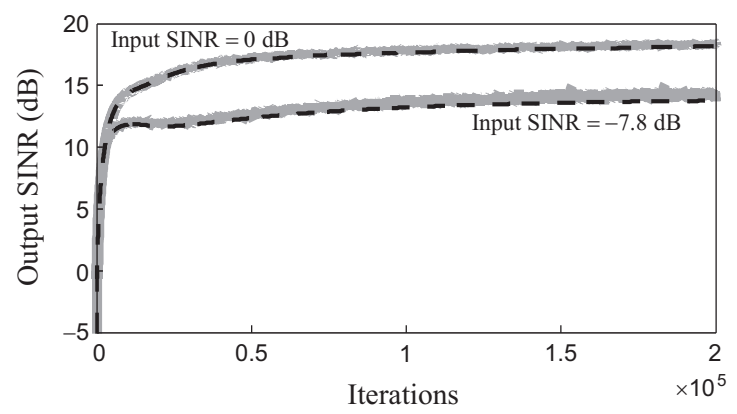

(c)

Fig. 2. Example 2. Results obtained by MC simulations (gray lines) and from the proposed model (dark-dashed lines). (a) Evolution of the real part of five weights of the beamforming vector. (b) Evolution of the imaginary part of five weights of the beamforming vector. (c) Evolution of the SINR. [The case of input SINR equal to $-7.8 \mathrm{~dB}$ has been omitted in (a) and (b) since very similar results are obtained.]

the SINR. Notice from these figures that the model predictions exhibit a very good match with the results obtained from the MC simulations irrespective of the azimuth spread $\Delta \theta_{k}$ [see Fig. 1(c)], the input SINR [see Fig. 2(c)], and the number of antennas in the array [compare Figs. 1 and 2], which is mainly because of the reduced number of simplifying assumptions used. So, since the model characterizes well the algorithm behavior, it can be used as a theoretical basis to study the algorithm without relying on MC simulations.

\section{CONCLUDING REMARKS}

In this paper, a stochastic model was derived describing the behavior of the KCLMS algorithm operating in an adaptive beamforming application. Specifically, model expressions were obtained for predicting the mean behavior of the beamforming vectors and the evolution of the SINR. Simulation results confirming the accuracy of the proposed model were shown and discussed. Further research could address the derivation of stability bounds for the step size and/or the development of model expressions characterizing the algorithm behavior in steady state.

\section{REFERENCES}

[1] E. Björnson, M. Bengtsson, and B. Ottersten, "Optimal multiuser transmit beamforming: A difficult problem with a simple solution structure," IEEE Signal Process. Mag., vol. 31, no. 4, pp. 142-148, Jul. 2014.

[2] S. Imani, S. A. Ghorashi, and M. Bolhasani, "SINR maximization in colocated MIMO radars using transmit covariance matrix," Signal Process., vol. 119, pp. 128-135, Feb. 2016.

[3] A. F. Molisch, V. V. Ratnam, S. Han, Z. Li, S. L. H. Nguyen, L. Li, and K. Haneda, "Hybrid beamforming for massive MIMO: A survey," IEEE Commun. Mag., vol. 55, no. 9, pp. 134-141, Sept. 2017.

[4] O. L. Frost, "An algorithm for linearly constrained adaptive array processing," Proc. IEEE, vol. 60, no. 8, pp. 926-935, Aug. 1972.

[5] D. R. Morgan, "Downlink adaptive array algorithms for cellular mobile communications," IEEE Trans. Commun., vol. 51, no. 3, pp. 476-488, Mar. 2003.

[6] J. E. Kolodziej, O. J. Tobias, R. Seara, and D. R. Morgan, "On the constrained stochastic gradient algorithm: Model, performance, and improved version," IEEE Trans. Signal Process., vol. 57, no. 4, pp. 1304-1315, Apr. 2009.

[7] R. de Lamare, L. Wang, and R. Fa, "Adaptive reduced-rank LCMV beamforming algorithms based on joint iterative optimization of filters: Design and analysis," Signal Process., vol. 90, no. 2, pp. 640-652, Feb. 2010.

[8] G. M. Zilli, C. A. Pitz, E. L. O. Batista, R. Seara, and W.-P. Zhu, "An adaptive approach for the joint antenna selection and beamforming optimization," IEEE Access, vol. 7, pp. 99 017-99 030, Aug. 2019.

[9] C. A. Pitz, E. L. O. Batista, R. Seara, and D. R. Morgan, "A novel approach for beamforming based on adaptive combinations of vector projections," Digit. Signal Process., vol. 97, ID 102621, Feb. 2020.

[10] L. C. Godara, Smart Antennas. Boca Raton, FL, USA: CRC Press, 2004.

[11] F. Rusek, D. Persson, B. K. Lau, E. G. Larsson, T. L. Marzetta, O. Edfors, and F. Tufvesson, "Scaling up MIMO: Opportunities and challenges with very large arrays," IEEE Signal Process. Mag., vol. 30 no. 1, pp. 40-60, Jan. 2013.

[12] Q. Nadeem, A. Kammoun, and M. Alouini, "Elevation beamforming with full dimension MIMO architectures in 5G systems: A tutorial," IEEE Commun. Surveys Tuts., vol. 21, no. 4, pp. 3238-3273, Jul. 2019.

[13] E. V. Kuhn, C. A. Pitz, M. V. Matsuo, K. J. Bakri, R. Seara, and J. Benesty, "A Kronecker product CLMS algorithm for adaptive beamforming," Digit. Signal Process., vol. 111, ID 102968, Apr. 2021.

[14] J. Benesty, I. Cohen, and J. Chen, Array Processing: Kronecker Product Beamforming. Cham, Zug, Switzerland: Springer, 2019.

[15] M. V. Matsuo and R. Seara, "On the stochastic analysis of the NLMS algorithm for white and correlated Gaussian inputs in time-varying environments," Signal Process., vol. 128, pp. 291-302, Nov. 2016.

[16] E. V. Kuhn, J. G. F. Zipf, and R. Seara, "On the stochastic modeling of a VSS-NLMS algorithm with high immunity against measurement noise," Signal Process., vol. 147, pp. 120-132, Jun. 2018.

[17] M. V. Matsuo, E. V. Kuhn, and R. Seara, "Stochastic analysis of the NLMS algorithm for nonstationary environment and deficient length adaptive filter," Signal Process., vol. 160, pp. 190-201, Jul. 2019.

[18] A. A. Falkovski, E. V. Kuhn, M. V. Matsuo, C. A. Pitz, E. L. O. Batista, and R. Seara, "Stochastic modeling of the CNLMS algorithm applied to adaptive beamforming," Signal Process., vol. 178, ID 107772, Jan. 2021.

[19] J. Benesty, C. Paleologu, and S. Ciochină, "On the identification of bilinear forms with the Wiener filter," IEEE Signal Process. Lett., vol. 24, no. 5, pp. 653-657, May. 2017.

[20] C. Paleologu, J. Benesty, and S. Ciochină, "Adaptive filtering for the identification of bilinear forms," Digit. Signal Process., vol. 75, pp. 153167, Apr. 2018.

[21] S. Haykin, Adaptive Filter Theory, 4th ed. Upper Saddle River, NJ, USA: Prentice Hall, 2002.

[22] A. H. Sayed, Adaptive Filters. Hoboken, NJ, USA: Wiley, 2009.

[23] C. Samson and V. Reddy, "Fixed point error analysis of the normalized ladder algorithm," IEEE Trans. Acoust., Speech, Signal Proc., vol. 31, no. 5, pp. 1177-1191, Oct. 1983.

[24] D. S. Bernstein, Matrix Mathematics: Theory, Facts, and Formulas, 2nd ed. Woodstock, OX, UK: Princeton University Press, 2009.

[25] R. W. Heath, N. González-Prelcic, S. Rangan, W. Roh, and A. M. Sayeed, "An overview of signal processing techniques for millimeter wave MIMO systems," IEEE J. Sel. Topics Signal Process., vol. 10, no. 3, pp. 436-453, Apr. 2016. 